\title{
Trial Inclusion Exclusion Criteria Version
}

National Cancer Institute

\section{Source}

National Cancer Institute. Trial Inclusion Exclusion Criteria Version. NCI Thesaurus. Code C83444.

A specific variant of the original trial inclusion exclusion criterion. 